\title{
Scheduling of Refinery Processes with Optimal Control Approach
}

\author{
HYUNGJIN PARK, JIN-KWANG BOK \\ AND SUNWON PARK \\ Department of Chemical Engineering, KAIST, \\ Taejon 305-701, Korea
}

Keywords: Optimal Control, Refinery Process, Scheduling, MILP Problem, Optimization

\begin{abstract}
A new scheduling algorithm based on an optimal control theory is presented for large-scale industrial problems. The main feature of this idea lies in a two-level hierarchical structure consisting of a reference model and a rigorous model, respectively. As a fair compromise between the excess of decision detail and long-range planning inspiration, we address simplified or relaxed models to derive optimal trajectories. The optimal trajectory is then used as a reference trajectory to be tracked in real time by a feedback control system to cope with uncertainty and modeling errors. We apply this idea to operation scheduling of refinery processes that convert crude oil mix into a variety of marketable products through a number of refining processes. The optimal control formulation is shown to enhance solution performance compared with the traditional mixed-integer model.
\end{abstract}

\section{Introduction}

Numerous scheduling methodologies for chemical process industries have been developed because the effective scheduling of operations has the potential for high economic return (Shah, 1998). Basically most of these methodologies are developed under assumption that operating parameters such as processing times and changeover times are deterministic for simplicity. However the inherent nature of the real processes is that the parameters vary slightly with operation. Hence the number of variables to be determined appears to be substantially large, even for small-scale realistic processes giving rise to computationally serious problems. The dimensionality can be quite reduced by aggregation, simplification, and approximation of scheduling models (Bok and Park, 1998). However, such algorithms cannot be a viable solution from the practitioner's point of view due to the fact that they do not leave enough flexibility to react to unpredictable events. In such context, the practitioners usually prefer scheduling methods based on the application of priority rules such as SPT (Shortest Processing Time) and EDD (Earliest Due Date) in spite of their myopic nature that results in poor quality performance.

As a fair compromise between the excess of decision detail and long-range planning inspiration, literature addresses an optimal control approach represented by a two-hierarchical structure consisting of a simplified or relaxed model to derive optimal trajectories and

Received on October 6, 2000. Correspondence concerning this article should be addressed to S. Park (E-mail address: swpark@mail.kaist.ac.kr). a rigorous model to keep the track of the trajectory (Brandimarte et al., 1995; Kogan and Khmelnitsky, 1996; Nott and Lee, 1999). A remarkable advantage of this approach is that computation is quite improved by coping with the uncertainty in the model. In this approach, acceptable control functions have been proposed that optimize the performance criteria, subject to the dynamic system constraints including all initial and final boundary conditions. The success of the optimal control approach is dependent on how close the reference trajectory can approximate the true optimal solution. To reduce the effect of modeling error a closed-loop feedback control has been applied. Nott and Lee (1999) have shown a new application area for optimal control, a sugar milling system that is a mixedbatch/continuous system.

The refinery industry has been a major innovator of optimization applications. Typically, crude oil scheduling involves ensuring continuity of the right type of crude oil to feed the main crude oil distillation units according to their monthly plan. Shah (1996) has shown the application of formal, mathematical programming techniques of scheduling crude oil supply. Lee et al. (1996) presented a MILP model over a uniformly discretized time horizon for the inventory management of refinery processes that import several types of crude oil delivered by different vessels. However their discrete representation of time horizon makes the models computationally expensive.

In this paper, we apply an optimal control algorithm to the short-term scheduling of refinery processes ranging from crude oil unloading to distillation charge.

This paper is organized as follows. First, we introduce the general optimal control theory. Next, prob- 


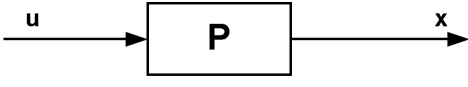

(a) Simple dynamic model

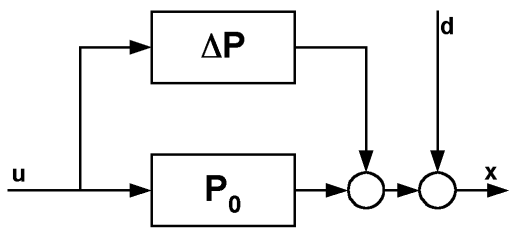

(b) Actual trajectory is affected by modeling error and uncertainty

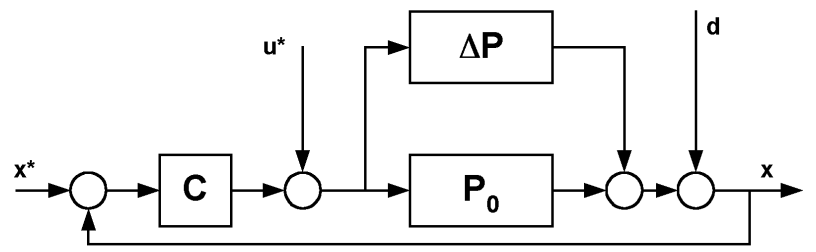

(c) Closed loop control system

Fig. 1 Closed loop control system

lem definition and mathematical formulation of the problem follow. And we describe the algorithm of presented the optimal control approach in detail. Then the numerical results of three examples are shown.

\section{Optimal Control Approach}

In a control-theoretic approach, a dynamic model of the manufacturing system should be developed in advance. For a continuous time domain, the dynamic model consists of a set of differential equations describing the time evolution of a state variable vector $\mathbf{x}(t)$ with a control vector $\mathbf{u}(t)$ :

$$
\frac{d \mathbf{x}(t)}{d t}=\mathbf{x}(t)=\mathbf{h}(\mathbf{x}(t), \mathbf{u}(t))
$$

Usually, both control and state vectors must comply with appropriate constraints:

$$
\mathbf{u}(t) \in \Omega ; \quad \mathbf{x}(t) \in \Psi
$$

Discrete time models arise when only time instants of the form $t=k \Delta(k=0,1,2, \ldots)$ are considered, where $\Delta$ is the discretization step.

For a given control input, a corresponding state trajectory is obtained by solving the state equations and the relation can be explained as shown in Fig. 1. Controlling the system requires finding a suitable con-

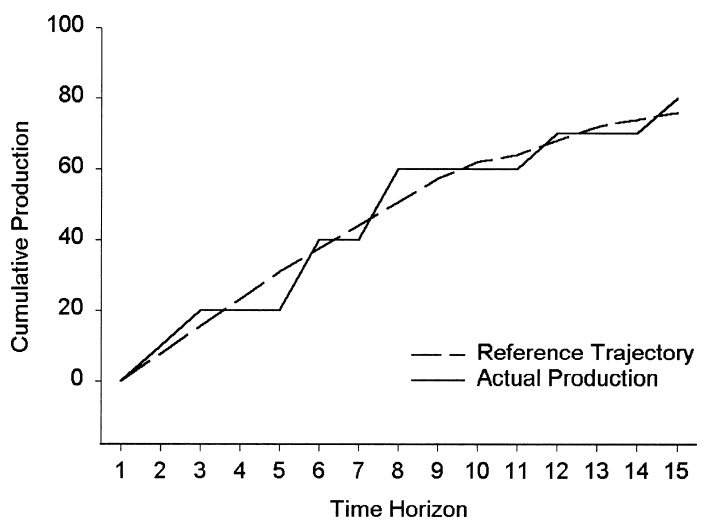

Fig. 2 Tracking target cumulative production trajectory

trol $\mathbf{u}(t)$ in order to obtain a satisfactory state trajectory. One way to accomplish this task is to define some performance measures (or objective function) $f$ and solve the following optimization problem:

$$
\min f(\mathbf{x}(t), \mathbf{u}(t))
$$

s.t.

$$
\begin{aligned}
& \mathbf{x}(t)=\mathbf{h}(\mathbf{x}(t), \mathbf{u}(t)) \\
& \mathbf{u}(t) \in \Omega \\
& \mathbf{x}(t) \in \Psi
\end{aligned}
$$

The solution of this optimization problem yields an optimal control $\mathbf{u}^{*}(t)$ and an optimal state trajectory $\mathbf{x}^{*}(t)$. When the state equations of model $\boldsymbol{P}$ are complex, the above optimization problem becomes very difficult to solve. A way to overcome this drawback is to solve the optimization problem only for a simplified representation $\boldsymbol{P}_{0}$ of the system, called the relaxed model. The state trajectory may be affected by disturbances $\mathbf{d}(t)$. For instance, an unpredictable demand rate plays the role of a disturbance. Therefore, the application of the optimal control $\mathbf{u}^{*}(t)$ from the nominal model $\boldsymbol{P}_{0}$ would not result in the optimal trajectory $\mathbf{x}^{*}(t)$.

In control theory, the approach to overcome these difficulties is the adoption of closed-loop or feedback control in Fig. 1. Instead of computing the optimal control $\mathbf{u}^{*}(t)$ and applying it to open-loop, i.e. neglecting real-time information on system performance, the optimal trajectory $\mathbf{x}^{*}(t)$ is used as a reference trajectory to be tracked.

In the past, optimal control has been applied to continuous production in a hierarchical two-level splitting of the optimization. At the upper hierarchical level the relaxed model of the manufacturing system is used, yielding a continuous flow schedule; the continuous flow schedule is an ideal production target, represented 


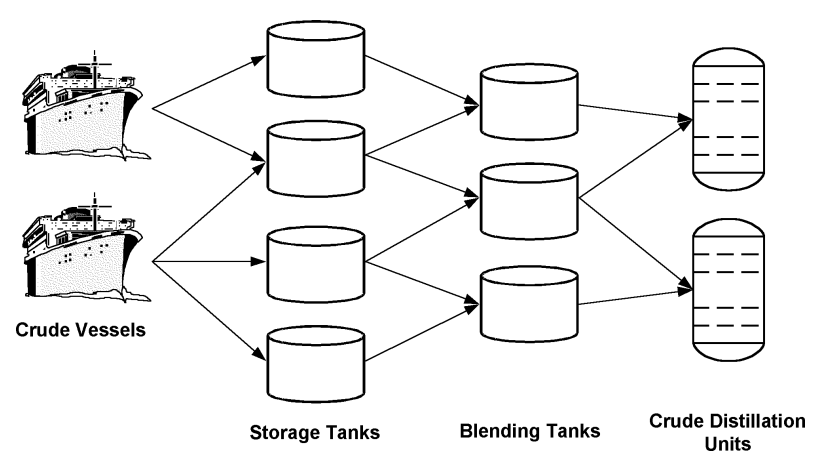

Fig. 3 Graphical overview of crude oil charging system

by a cumulative production trajectory. And at the lower level, detailed schedules are computed, using accurate models that account for all the features of the production system, with the objective of following the targets proposed by the higher level. Figure 2 shows the procedure of tracking a target production trajectory. This is essentially the problem of tracking a reference signal in a feedback control system and can be accomplished off-line or in real time.

\section{Problem Definition}

The main role of refining operation is to transfer crude oil to a storage tank in a refinery plant, and to convert crude oil mix into a variety of marketable products through a number of refining processes. The system configuration of this scheduling problem corresponds to a multistage system consisting of vessels, storage tanks, blending tanks, and CDUs (crude distillation unit) as illustrated in Fig. 3.

During a given scheduling horizon, crude vessels arrive in the vicinity of the refinery docking station and, if busy, wait for unloading of the preceding vessel in the docking station. At the docking station, crude oil is unloaded into storage tanks. Crude oil is then transferred from storage tanks to blending tanks that are buffers to produce a crude mix, of which component compositions are determined at the planning level. The crude oil mix in each blending tank is then charged into a CDU.

The followings are operating rules that have to be obeyed in this problem (Lee et al., 1996).

- If a vessel does not arrive at the wharf, it cannot unload the crude.

- If a vessel leaves the wharf, it cannot unload the crude oil.

- The vessel should leave the wharf after its arrival.

- The vessel in the sea cannot arrive at the wharf if the preceding vessel does not leave.

- While a blending tank is charging CDU, crude cannot be fed into the blending tank and vice versa.
- CDU cannot be charged simultaneously by different blends.

The major operating constraints are as follows:

- Equipment capacity limitations (tank capacity, pumping rate).

- Quality limitations on each mixed crude oil.

- Demand of each mixed oil to be charged into CDU.

\section{Model Formulation}

There are some assumptions for the model that will be proposed in this paper:

- Changeover times on the CDU are neglected.

- There is perfect mixing in the blending tanks.

- Additional mixing time is not required before it charges CDU.

- Only specific key components in crude or mixed oil decide the property of each oil.

- $\quad$ Each vessel delivers the specific oil from a specific source of supply to an assigned storage tank.

And the proposed scheduling model is based on a uniform discretization of time in the given scheduling horizon.

The objective of this problem is to maximize the operating profit from the defined refinery processes. The total profit of the crude oil production can be expressed as the subtraction of operating cost from the revenue of the final oil mixture.

The operating cost consists of the unloading cost from crude vessels to storage tanks, the vessel waiting cost in the sea due to the previously docked vessel in the docking station, the inventory cost of storage and blending tanks, and the changeover cost by transition of connection between blending tanks and CDUs.

Therefore, the objective function can be expressed as follows: $($ Profit $)=($ sales revenue $)-($ unloading cost for the crude vessel) - (demurrage cost of vessels) (inventory cost for storage and blending tanks) (changeover cost in CDUs).

\section{Maximize}

$$
\begin{aligned}
\text { PROFIT }= & \sum_{j=1}^{J} \operatorname{PRICE}_{j} \cdot T O T_{\mathrm{prod}, j} \\
& -\sum_{v=1}^{V} C_{\mathrm{unload}, v}\left(T_{\mathrm{L}, v}-T_{\mathrm{F}, v}\right) \\
& -\sum_{v=1}^{V} C_{\mathrm{stay}, v}\left(T_{\mathrm{F}, v}-T_{\mathrm{ARR}, v}\right) \\
& -\sum_{i=1}^{I} \sum_{t=1}^{T} C_{\mathrm{inv} \_\mathrm{ST}, i}\left(\frac{V_{\mathrm{S}, i, t}+V_{\mathrm{S}, i, t-1}}{2}\right)
\end{aligned}
$$




$$
\begin{aligned}
& -\sum_{j=1}^{J} \sum_{t=1}^{T} C_{\text {inv_BT, } j}\left(\frac{V_{\mathrm{B}, j, t}+V_{\mathrm{B}, j, t-1}}{2}\right) \\
& -\sum_{t=1}^{T} \sum_{j=1}^{J} \sum_{j^{\prime}=1}^{J} \sum_{l=1}^{L} C_{\text {setup }, j, j^{\prime}, l} Z_{j, j^{\prime}, l, t}
\end{aligned}
$$

Unloading cost for the crude vessel is proportional to the unloading duration of vessels at the docking station and vessel waiting cost is proportional to the time difference between the unloading start time and arrival time. Tank inventory costs are calculated according to the trapezoid area and changeover cost is proportional to the number of the crude mix switch (Lee et al., 1996).

\section{Subject to:}

a. Vessel arrival and departure operation rules.

Each vessel arrives and leaves the docking station for unloading only once.

$$
\sum_{t=1}^{T} X_{\mathrm{F}, v, t}=1, \sum_{t=1}^{T} X_{\mathrm{L}, v, t}=1 \quad \forall v
$$

Unloading starting time and completion time can be calculated by following equations.

$$
T_{\mathrm{F}, v}=\sum_{t=1}^{T} t X_{\mathrm{F}, v, t}, \quad T_{\mathrm{L}, v}=\sum_{t=1}^{T} t X_{\mathrm{L}, v, t} \quad \forall v
$$

Each crude vessel should start unloading after arrival time set and cannot arrive at the docking station for unloading unless the preceding vessel leaves.

$$
T_{\mathrm{F}, v} \geq T_{\mathrm{ARR}, v}, \quad T_{\mathrm{F}, v+1} \geq T_{\mathrm{L}, v} \quad \forall v
$$

Each crude vessel needs not less than the minimum duration time of the vessel.

$$
T_{\mathrm{L}, v}-T_{\mathrm{F}, v} \geq \operatorname{DURATION}_{v} \quad \forall v
$$

Unloading is possible between unloading starting time and completion time.

$$
X_{\mathrm{W}, v, t} \leq \sum_{m=1}^{t} X_{\mathrm{F}, v, m}, \quad X_{\mathrm{W}, v, t} \leq \sum_{m=t}^{T} X_{\mathrm{L}, v, m} \quad \forall v
$$

b. Material balance equations for the vessel.

Crude oil in vessel $v$ at time $t=$ initial crude oil in the vessel $v$-crude oil transferred from vessel $v$ to storage tanks up to time $t$.

$$
V_{\mathrm{V}, v, t}=V_{\mathrm{V}, v, 0}-\sum_{i=1}^{I} \sum_{m=1}^{t} F_{\mathrm{VS}, v, i, m} \quad \forall v, t
$$

Operating constraints are needed on crude oil transfer rate from vessel $v$ to storage tank $i$ at time $t$.

$$
F_{\mathrm{VS}, v, i, \min } X_{\mathrm{W}, v, t} \leq F_{\mathrm{VS}, v, i, t} \leq F_{\mathrm{VS}, v, i, \max } X_{\mathrm{W}, v, t} \quad \forall v, i
$$

The volume of crude oil transferred from vessel $v$ to storage tanks during the scheduling horizon equals to the initial crude oil volume of vessel $v$.

$$
\sum_{i=1}^{I} \sum_{t=1}^{T} F_{\mathrm{VS}, v, i, t}=V_{\mathrm{V}, v, 0} \quad \forall v
$$

c. Material balance equations for the storage tank.

Crude oil in storage tank $i$ at time $t=$ initial crude oil in storage tank $i+$ crude oil transferred from vessels to storage tank $i$ up to time $t$ - crude oil transferred from storage tank $i$ to blending tanks up to time $t$.

$$
V_{\mathrm{S}, i, t}=V_{\mathrm{S}, i, 0}+\sum_{v=1}^{V} \sum_{m=1}^{t} F_{\mathrm{VS}, v, i, m}-\sum_{j=1}^{J} \sum_{m=1}^{t} F_{\mathrm{SB}, i, j, m} \quad \forall i, t
$$

Operating constraints are needed on crude oil transfer rate from storage tank $i$ to blending tank $j$ at time $t$.

The term $1-\sum_{l=1}^{L} D_{j, l, t}$ denotes that if blending tank $j$ is charging any CDU, there is no oil transfer from storage tank $i$ to blending $\operatorname{tank} j$.

$$
\begin{aligned}
F_{\mathrm{SB}, i, \min }\left(1-\sum_{l=1}^{L} D_{j, l, t}\right) \leq F_{\mathrm{SB}, i, j, t} \\
\leq F_{\mathrm{SB}, i, j, \max }\left(1-\sum_{l=1}^{L} D_{j, l, t}\right)
\end{aligned}
$$

Storage tank $i$ has volume capacity at time $t$.

$$
V_{\mathrm{S}, i, \min } \leq V_{\mathrm{S}, i, t} \leq V_{\mathrm{S}, i, \max } \quad \forall i \in I
$$

Cumulative flow amount from storage tank $i$ to blending $\operatorname{tank} j$ at time $t$ can be formulated by the sum- 
mation of crude oil transferred from storage tank $i$ to blending tanks up to time $t$.

$$
C F_{\mathrm{SB}, i, j, t}=C F_{\mathrm{SB}, i, j, t-1}+F_{\mathrm{SB}, i, j, t} \quad \forall i, j, t
$$

d. Material balance equations for the blending tank.

Crude oil mix in blending $\operatorname{tank} j$ at time $t=$ initial mixed oil in blending tank $j+$ crude oil transferred from storage tanks to blending tank $j$ up to time $t$-crude oil mix $j$ charged into CDUs up to time $t$.

$$
\begin{array}{r}
V_{\mathrm{B}, j, t}=V_{\mathrm{B}, j, 0}+\sum_{i=1}^{I} \sum_{m=1}^{t} F_{\mathrm{SB}, i, j, m}-\sum_{l=1}^{L} \sum_{m=1}^{t} F_{\mathrm{BC}, j, l, m} \\
\forall j, l, t
\end{array}
$$

Operating constraints on mixed oil transfer rate from blending tank $j$ to CDU $l$ at time $t$.

$$
F_{\mathrm{BC}, j, l, \min } D_{j, l, t} \leq F_{\mathrm{BC}, j, l, t} \leq F_{\mathrm{BC}, j, l, \max } D_{j, l, t} \quad \forall j, l, t
$$

Blending tank $j$ has volume capacity at time $t$.

$$
V_{\mathrm{B}, j, \min } \leq V_{\mathrm{B}, j, t} \leq V_{\mathrm{B}, j, \max } \quad \forall j, t
$$

Total production amount of crude oil mix $j$ should be larger than the demand of crude mix $j$ for the scheduling horizon.

$$
\sum_{l=1}^{L} \sum_{t=1}^{T} F_{\mathrm{BC}, j, l, t} \geq \operatorname{DEMAND}_{j} \quad \forall j
$$

Cumulative flow amount from blending tank $j$ to CDU $l$ at time $t$ can be formulated by the summation of crude oil transferred from blending $\operatorname{tank} j$ to CDU $l$ up to time $t$.

$$
C F_{\mathrm{BC}, j, i, t}=C F_{\mathrm{BC}, j, l, t-1}+F_{\mathrm{BC}, j, l, t} \quad \forall j, l, t
$$

e. Material balance equations for component $k$ in the blending tank.

Volume of component $k$ in blending tank $j$ at time $t=$ initial component $k$ in blending $\operatorname{tank} j+$ component $k$ in crude oil transferred from storage tanks to blending tank $j$ up to time $t$ - component $k$ in crude oil mix $j$ transferred to CDUs up to time $t$.

$$
\begin{array}{r}
v_{\mathrm{B}, j, t}=v_{\mathrm{B}, j, 0}+\sum_{m=1}^{t}\left(\sum_{i=1}^{I} f_{\mathrm{SB}, i, j, m}-\sum_{l=1}^{L} f_{\mathrm{BC}, j, l, m}\right) \\
\forall j, k, t \quad(\mathrm{e} 1)
\end{array}
$$

Operating constraints on volumetric flow rate of component $k$ from storage tank $i$ to blending tank $j$

$$
f_{\mathrm{SB}, i, j, k, t}=F_{\mathrm{SB}, i, j, t} \xi_{\mathrm{S}, i, k} \quad \forall j, k
$$

Operating constraints on volumetric flow rate of component $k$ from blending tank $j$ to $\mathrm{CDU} l$

$$
\begin{array}{r}
F_{\mathrm{BC}, j, l, t} \xi_{\mathrm{B}, j, k, \min } \leq f_{\mathrm{BC}, j, l, k, t} \leq F_{\mathrm{BC}, j, l, t} \xi_{\mathrm{B}, j, k, \max } \\
\forall j, k, l, t
\end{array}
$$

Volume capacity limitations for component $k$ in blending $\operatorname{tank} j$ at time $t$

$$
V_{\mathrm{B}, j, t} \xi_{\mathrm{B}, j, k, \min } \leq v_{\mathrm{B}, j, k, t} \leq V_{\mathrm{B}, j, t} \xi_{\mathrm{B}, j, k, \max } \quad \forall j, k, l, t
$$

f. Operating rules for crude oil charging.

Blending tank $j$ can charge at most one CDU at any time $t$ and CDU $l$ can be charged at most by one blending tank at any time $t$.

$$
\sum_{l=1}^{L} D_{j, l, t} \leq 1, \sum_{j=1}^{J} D_{j, l, t} \leq 1 \quad \forall j, l, t
$$

If CDU $l$ is charged by crude oil mix $j$ at time $t-$ 1 and charged by $j^{\prime}$ at time $t$, changeover cost is involved.

$$
Z_{j, j^{\prime}, l, t} \geq D_{j^{\prime}, l, t}+D_{j, l, t-1}-1 \quad \forall j, j^{\prime}\left(j \neq j^{\prime}\right), l, t
$$

\section{Scheduling with Optimal Control Approach}

In the optimal control approach, we often use a simplified or relaxed model to derive an optimal trajectory, which is then used as a reference trajectory to be tracked in real time by a feedback control system.

\subsection{First step: Relaxed model and reference trajec- tory}

For production scheduling problems, a suitable relaxed model may be obtained by approximating the discrete material flow as a continuous flow for simplicity. By the operating rules, this MILP problem has the following binary variables, such as $X_{\mathrm{F}, v, t}, X_{\mathrm{L}, v, t}$, and $D_{j, l, t} . X_{\mathrm{F}, v, t}$ and $X_{\mathrm{L}, v, t}$ are used to determine the unloading time of crude vessels and $D_{j, l, t}$ is introduced to denote the operating rule that each tank can feed at most one CDU at one time interval and each CDU is charged by only one mixed crude oil at one time interval. The number of these binary variables is so large that the corresponding MILP model becomes computationally very expensive. So we can simplify the complex MILP problem by relaxing the binary variables. 
We then add the bounds for $D_{j, l, t}$ as follows:

$$
0 \leq D_{j, l, t} \leq 1, \quad \forall j, l, t
$$

Therefore example 1 is relaxed to an LP model which is relatively easy to solve. In the cases of example 2 and 3, we need additional binary variables, $X_{\mathrm{F}, v, t}$ and $X_{\mathrm{L}, v, t}$ and should solve the resulting MILP problem. The cumulative production curve from the relaxed model and the complete model may appear different. However, comparing with the traditional MILP formulation, there is no change of the objective function. In the relaxed model, we assume the crude oil flows between tanks are continuous flows. Assuming $D_{j, l, t}$ are continuous varables (after formulating relaxed model), we can obtain the reference trajectory by solving the relaxed model.

\subsection{Second step: Tracking reference trajectory}

After obtaining the reference trajectory from the relaxed model, the complete scheduling problem must be solved. This can be done in different ways. A simple approach is to use the reference trajectory in order to define part priorities. The actual processing scheduling is selected in order to minimize the error between continuous flow and real cumulative production trajectories. This can be accomplished by using the simple greedy strategy adopted in the Toyota Goal Chasing method (Miltenburd, 1989). The objective might be one of the following in this method.

$$
\begin{array}{ll}
\text { Minimize } & \sum_{j}^{J} \sum_{i}^{I}\left(T_{\mathrm{ACTUAL}, i, j}-T_{\mathrm{REF}, i, j}\right)^{2} \\
\text { or minimize } & \sum_{j}^{J} \sum_{i}^{I}\left|T_{\mathrm{ACTUAL}, i, j}-T_{\mathrm{REF}, i, j}\right|
\end{array}
$$

In Eq. (7), $T_{\mathrm{REF}, i, j}$ is reference trajectory from unit $i$ to unit $j$ and $T_{\mathrm{ACTUAL}, i, j}$ is the solution solved by the optimization problem with constraints, or the tracking trajectory. These objective functions seek to minimize the variation (either squared deviations or absolute deviations) of actual production from the desired production. Miltenburd (1989) and Nott (1998) used the cumulative production as the reference trajectory.

In the case of refinery crude oil processing, the crude oil flow procedure is very complex due to many storage and blending tanks and operation rules. If the cumulative flows of the whole transport lines are used as the reference trajectories, the complete MILP problem requires much computation time. Therefore, instead of using the cumulative flow, we use the binary variables, $D_{j, l, t}, X_{\mathrm{F}, v, t}$ and $X_{\mathrm{L}, v, t}$ as the reference trajectories.

In this system, the binary variable $D_{j, l, t}$ plays an important role of the crude oil transport scheduling.

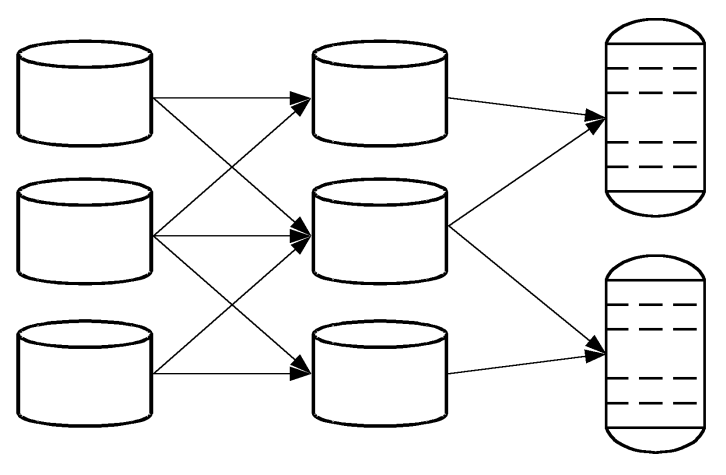

3 Storage Tanks

3 Blending Tanks

2 Crude Distillation Units

Fig. 4 Crude oil charging system of example 1

So if it is determined, whole scheduling can be obtained.

Tracking the reference trajectory is accomplished by penalizing any deviations from the reference trajectories of $D_{\mathrm{REF}, j, t, t}, X_{\mathrm{F}, \mathrm{REF}, \mathrm{v}, t}$ and $X_{\mathrm{L}, \mathrm{REF}, \mathrm{t},}$. Therefore, the new objective function consists of penalty terms of binary variable deviations.

$$
\begin{aligned}
\text { PROFIT }= & \sum_{j=1}^{J} \text { PRICE }_{j} \cdot \text { TOT } \\
& - \text { PEN }, j_{\mathrm{D}} \sum_{t=1}^{T} \sum_{l=1}^{L} \sum_{j=1}^{J}\left|D_{j, l, t}-D_{\mathrm{REF}, j, l, t}\right| \\
& -P E N T_{\mathrm{XF}} \sum_{t=1}^{T} \sum_{v=1}^{V}\left|X_{\mathrm{F}, v, t}-X_{\mathrm{F}, \mathrm{REF}, v, t}\right| \\
& - \text { PENT }_{\mathrm{XL}} \sum_{t=1}^{T} \sum_{v=1}^{V}\left|X_{\mathrm{L}, v, t}-X_{\mathrm{L}, \mathrm{REF}, v, t}\right|
\end{aligned}
$$

(Example 1 does not have the third term, $X_{\mathrm{F}, \mathrm{t}, \mathrm{p}}$ penalty and fourth term, $X_{\mathrm{L}, v, t}$ penalty.)

We can obtain the tracking results by solving the complete MILP problem with new objective function. The tracking solution in this step is near the reference trajectory solution from the relaxed model. It reduces the number of iterations of lots of branches by giving penalties to the objective function in order to track the reference trajectories. As the result, we can obtain the solution with less calculation time.

In this system, we use the bigger values for $P E N T_{\mathrm{XF}}$ and $P E N T_{\mathrm{XL}}$ than the value for $P E N T_{\mathrm{D}}$. If the penalty values for $X_{\mathrm{F}, v, t}$ and $X_{\mathrm{L}, v, t}$ are bigger than that for $D_{j, l, t}$, the crude oil unloading time is fixed close to the reference determined by the relaxed model. After fixing $X_{\mathrm{F}, v, t}$ and $X_{\mathrm{L}, v, t}$, we find the optimum $D_{j, l, t}$. 
Table 1 System information for example 1

\begin{tabular}{|c|c|c|c|c|c|}
\hline \multicolumn{3}{|c|}{ Scheduling horizon (\# of unit time) } & \multicolumn{3}{|c|}{10} \\
\hline Storage tanks & Capacity & \multicolumn{2}{|c|}{ Initial oil amount } & Component 1 & Component 2 \\
\hline Tank 1 & 100 & & 100 & 0.01 & 0.04 \\
\hline Tank 2 & 100 & & 80 & 0.03 & 0.02 \\
\hline Tank 3 & 100 & & 60 & 0.05 & 0.01 \\
\hline \multicolumn{3}{|c|}{ Number of blending tanks } & \multicolumn{3}{|c|}{3} \\
\hline Blending tanks & Capacity & & oil amount & $\begin{array}{l}\text { Initial comp. } 1 \\
\text { (min, } \max )\end{array}$ & $\begin{array}{l}\text { Initial comp. } 2 \\
\text { (min, } \max )\end{array}$ \\
\hline Tank 1 & 100 & & 30 & $0.0167(0.01,0.02)$ & $0.0333(0.03,0.038)$ \\
\hline Tank 2 & 100 & & 50 & $0.03(0.025,0.035)$ & $0.023(0.018,0.027)$ \\
\hline Tank 3 & 100 & & 40 & $0.0433(0.04,0.048)$ & $0.0133(0.01,0.018)$ \\
\hline \multicolumn{3}{|c|}{ Number of CDU } & \multicolumn{3}{|c|}{2} \\
\hline \multicolumn{3}{|c|}{ Tank inventory unit costs } & \multicolumn{3}{|c|}{$\begin{array}{l}\text { Storage tank }(1: 0.06,2: 0.05,3: 0.04) \\
\text { Blending tank }(1: 0.09,2: 0.08,3: 0.07)\end{array}$} \\
\hline \multicolumn{3}{|c|}{ Turnover produced } & \multicolumn{3}{|c|}{ Oil mix $(1: 100,2: 90,3: 80)$} \\
\hline \multicolumn{3}{|c|}{ Unit changeover cost for charged oil switch } & \multicolumn{3}{|c|}{50 (independent of sequence and CDU) } \\
\hline \multicolumn{3}{|c|}{ Demand of mixed oils by CDUs } & \multicolumn{3}{|c|}{ Oil mix $(1: 100,2: 100,3: 100)$} \\
\hline
\end{tabular}

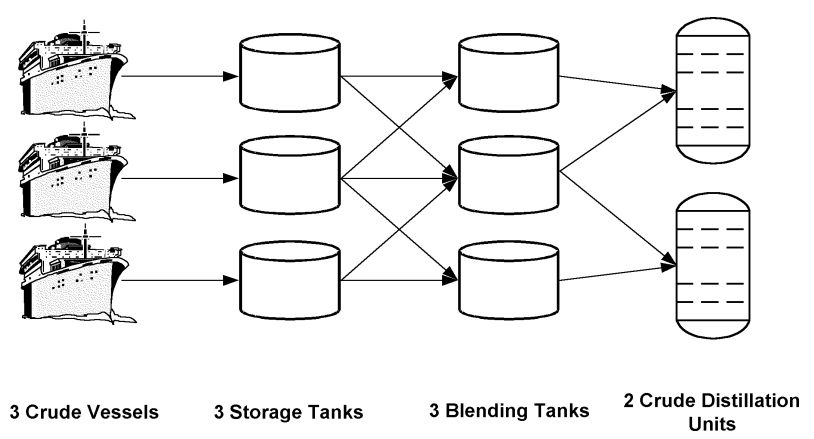

Fig. 5 Crude oil charging system of example 2

\section{Examples}

In this paper, we have three examples. The first is for the inventory management of storage tanks and blending tanks and the second example includes crude unloading from crude oil vessel. In the both examples, we assume 3 storage tanks and 3 blending tanks. Example 3 deals with an industry size problem that has 3 crude vessels, 6 storage tanks, 4 blending tanks and 3 CDUs.

The modeling system GAMS (Brooke et al., 1992) is used for setting up the optimization models and the problems are solved by OSL on a SUN SPARC 10.

\subsection{Example 1}

Figure 4 illustrates the system for example 1. The scheduling horizon for this problem is 10 time inter-

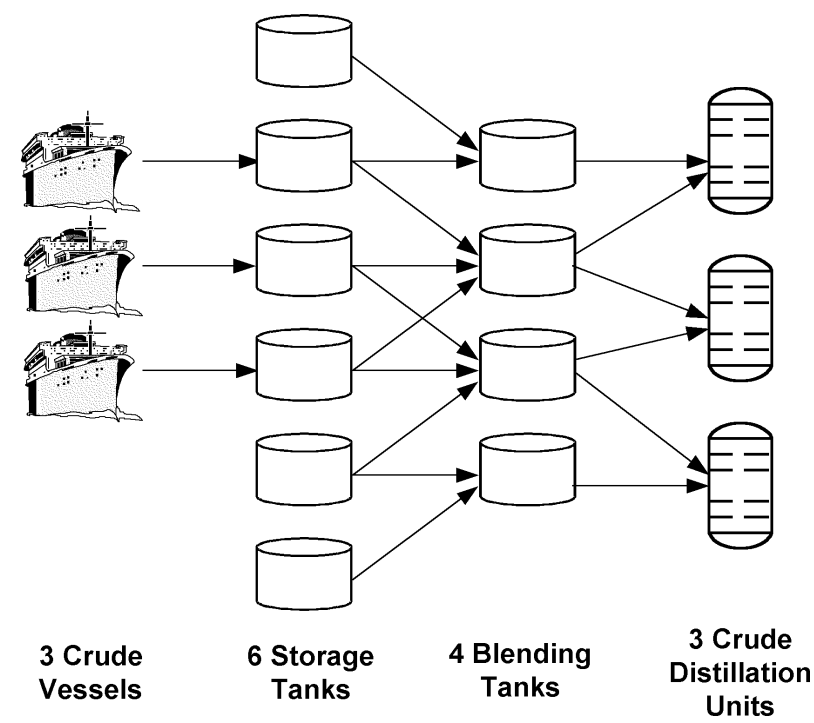

Fig. 6 Crude oil charging system of example 3

vals, and initially there is 100 units of crude oil in storage tank 1, 80 units in storage tank 2, and 60 units in storage tank 3 . Blending tank 1 contains crude 1 and crude 2 in its crude mix 1 . Crude mix 2 is composed of 1,2 , and 3 , while blending tank 3 contains crude 2 and 3. CDU 1 is fed by blending tank 1 and 2, and CDU 2 is blending tank 2 and 3. The two key components in crude and mixed oil should be considered. The tank inventory unit costs are different from each other and 
Table 2 System information for example 2

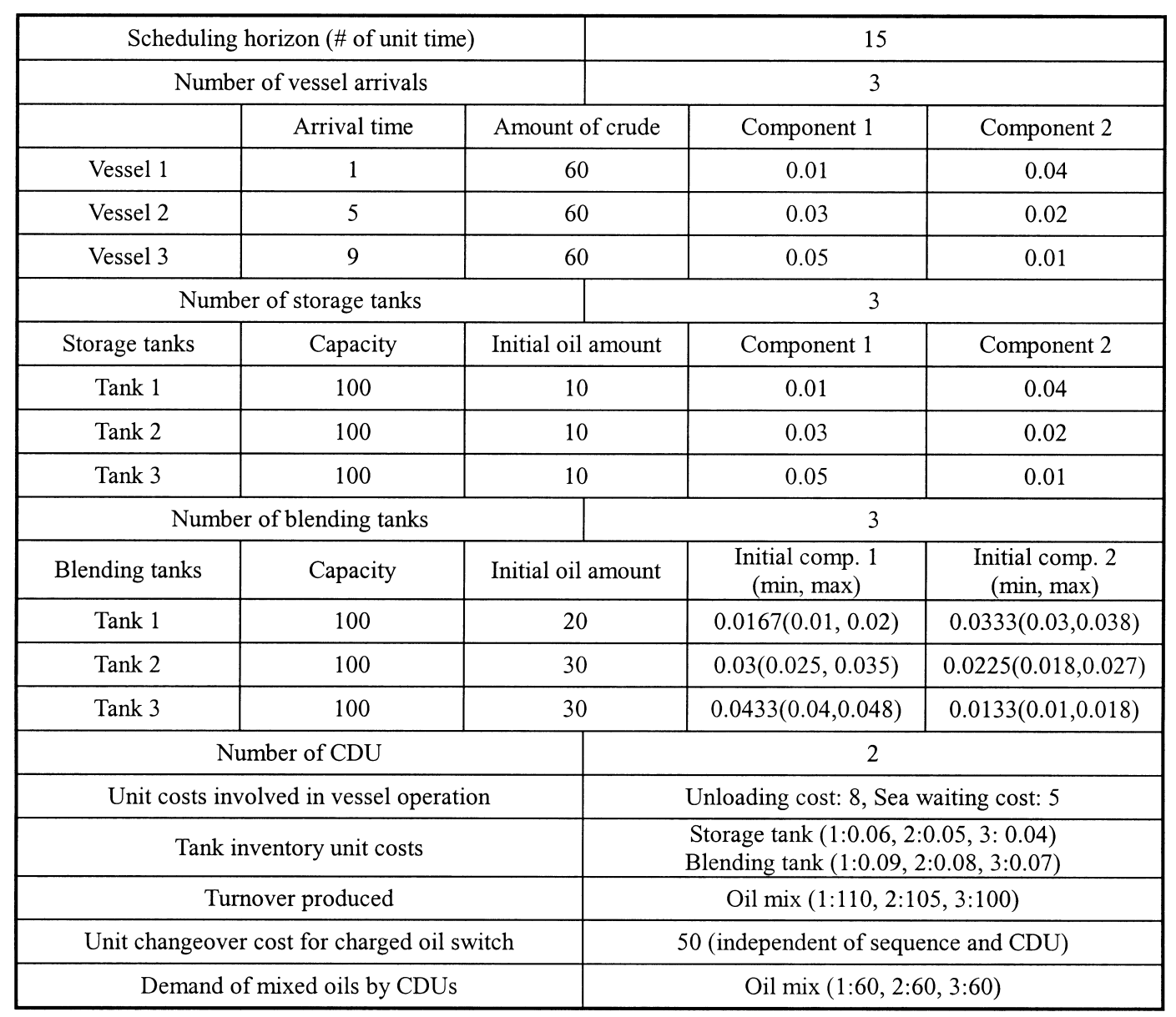

turnover of crude mix is 100 units for crude mix 1,90 units for crude mix 2 , and 80 units for crude mix 3 . The minimum demand by CDUs for the given scheduling horizon is 100 for each crude mix. Table 1 shows the data involved in this problem.

\subsection{Example 2}

Example 2 includes crude unloading from crude oil vessels in Fig. 5. The scheduling horizon for this problem is 15 time intervals, and three crude vessels arrive at times 1, 5 and 9, respectively. Crude vessel 1 delivers crude 1 to storage tank 1 , crude vessel 2 to storage tank 3 , and crude vessel 3 to storage tank 2 . Each crude vessel contains 60 amount of crude oil. Unloading cost of each vessel is 8 and demurrage cost is 5. The minimum demand by CDUs for the given scheduling horizon is 60 for each crude mix. Table 2 shows the data involved in this problem.

\subsection{Example 3}

Example 3 is the oil flow network of industry size problem in Fig. 6. This example consists of 3 crude vessels, 6 storage tanks, 4 blending tanks, and 3 CDUs. Three crude vessels arrive at times 1, 6 and 11, respectively and vessel 1 unloads oil to storage tank 2 , vessel 2 to storage tank 3 , vessel 3 to storage tank 4 . Table 3 gives the data of example 3 .

\section{Results}

Table 4 compares the computational results of the traditional MILP formulation with the optimal control algorithm proposed in this paper. In the case of traditional MILP formulation of all examples, the problem is solved with $1 \%$ tolerance to the optimum.

Figures 7-13 show the reference trajectories and tracking trajectories of crude oil flows from the blending tanks to CDUs. They are the results from the tracking trajectory of $D_{j, l, t}$ obtained in the second step. In the case of the reference trajectory, with assumption that each crude oil transfer line is contiuous flow, the problem is solved. So the cumulative flow increases continuously linearly. On the other hand, the tracking trajectories of complete MILP formulation show step change due to the characteristics of operation rules. The solutions obtained with optimal control approach track well to the reference trajectories.

The solution obtained by the proposed optimal control algorithm shows some deviation from the global optimum (less than $1 \%$ ). However, the proposed algorithm shows excellent performance for a complex problem that has been shown to be very difficult to solve. In other words, while the solution can not guar- 
Table 3 System information for example 3

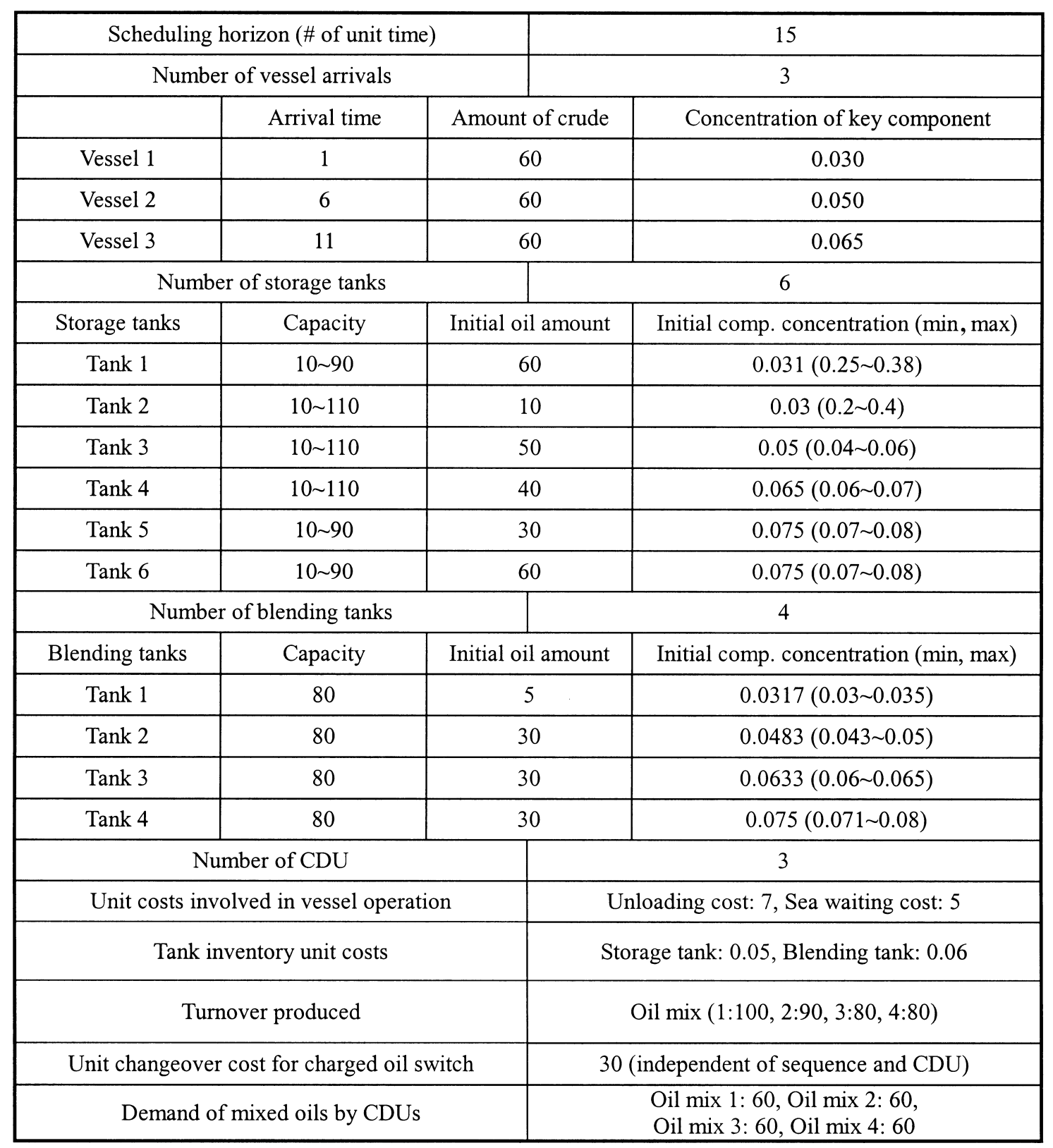

Table 4 Computational results

\begin{tabular}{|c|c|c|c|c|c|c|c|c|}
\hline & & Constraints & $\begin{array}{c}\text { Con } \\
\text { Var }\end{array}$ & $\begin{array}{l}\text { nuous } \\
\text { ables }\end{array}$ & $\begin{array}{c}0-1 \\
\text { Variables }\end{array}$ & $\begin{array}{c}\text { CPU time } \\
{[\mathrm{s}]}\end{array}$ & Iterations & $\begin{array}{c}\text { Optimal } \\
\text { Solutions }\end{array}$ \\
\hline \multirow[t]{3}{*}{ Ex. 1} & Original MILP & 813 & & 442 & 40 & 923.1 & 78,494 & $32,687.8$ \\
\hline & Proposed Method & 813 & 1 step: & 482 & 0 & 5.7 & 590 & $32,525.5$ \\
\hline & & & 2 step: & 503 & 40 & 44.7 & 4,813 & \\
\hline \multirow[t]{3}{*}{ Ex. 2} & Original MILP & 1,352 & & 739 & 96 & $4,653.3$ & 274,721 & $26,966.8$ \\
\hline & Proposed Method & 1,352 & 1 step: & 797 & 36 & 24.3 & 1,278 & $26,782.4$ \\
\hline & & & 2 step: & 918 & 96 & 133.4 & 7,451 & \\
\hline \multirow[t]{3}{*}{ Ex. 3} & Original MILP & 2,397 & & 1,133 & 120 & $6,682.7$ & 237,463 & $38,079.2$ \\
\hline & Proposed Method & 2,397 & 1 step: & 1,223 & 30 & 66.5 & 3,247 & $37,646.8$ \\
\hline & & & 2 step: & 1,404 & 120 & 244.5 & 11,001 & \\
\hline
\end{tabular}


(a) Tracking reference trajectory from $\mathrm{BT}(1)$ to $\mathrm{CDU}(1)$

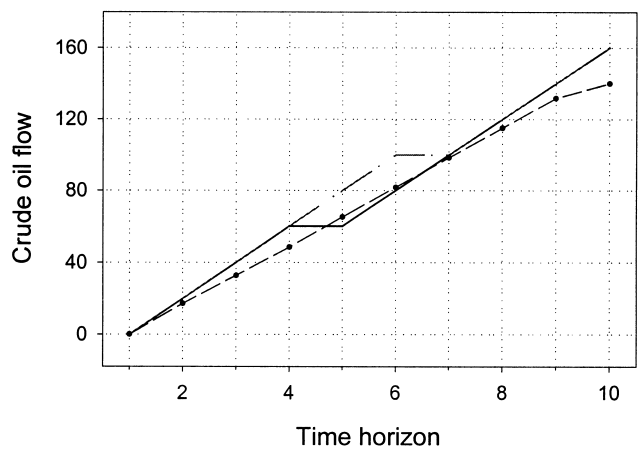

(b) Tracking reference trajectory from $\mathrm{BT}(2)$ to $\mathrm{CDU}(1)$

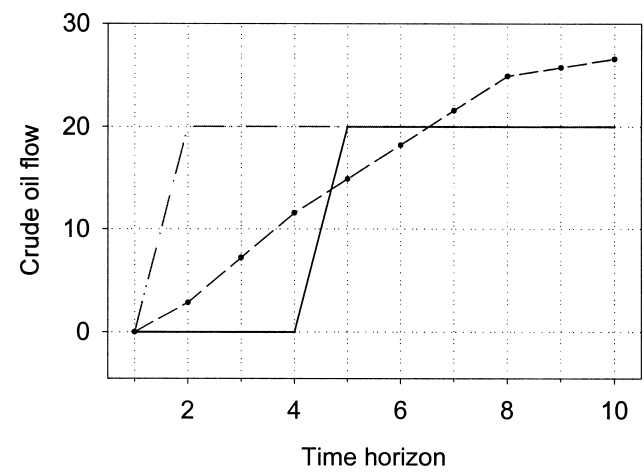

Fig. 7 Tracking reference trajectory from blending tank to CDU (example 1(a), (b)). Dot line: reference trajectory, real line: tracking trajectory, dash-dot line: original MILP trajectory

(c) Tracking reference trajectory from $\mathrm{BT}(2)$ to $\mathrm{CDU}(2)$

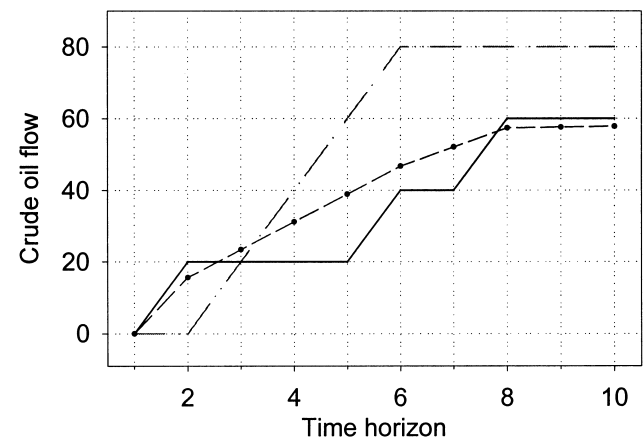

(d) Tracking reference trajectory from BT(3) to $\mathrm{CDU}(2)$

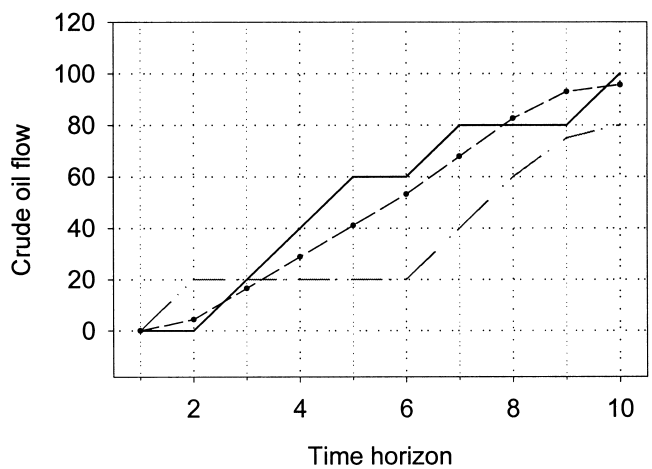

Fig. 8 Tracking reference trajectory from blending tank to CDU (example 1(c), (d)) (a) Tracking reference trajectory from $\mathrm{BT}(1)$ to $\mathrm{CDU}(1)$

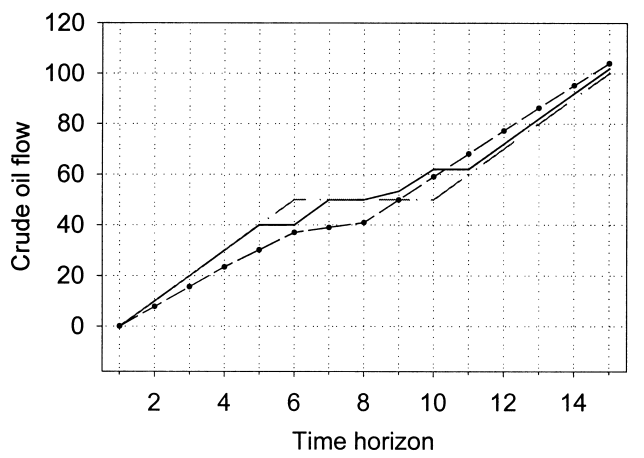

(b) Tracking reference trajectory from $\mathrm{BT}(2)$ to $\mathrm{CDU}(1)$

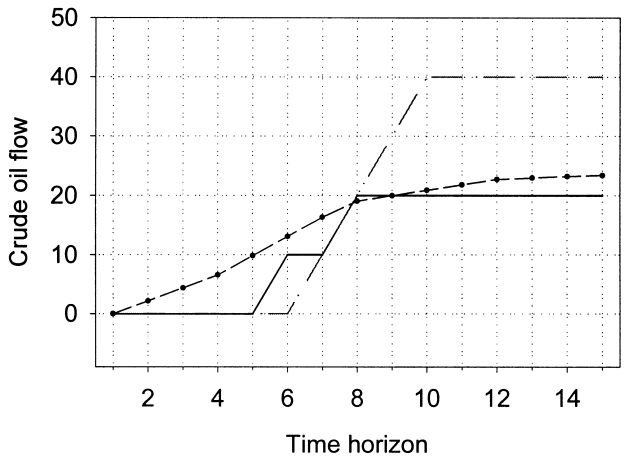

Fig. 9 Tracking reference trajectory from blending tank to CDU (example 2(a), (b))

(c) Tracking reference trajectory from $\mathrm{BT}(2)$ to $\mathrm{CDU}(2)$

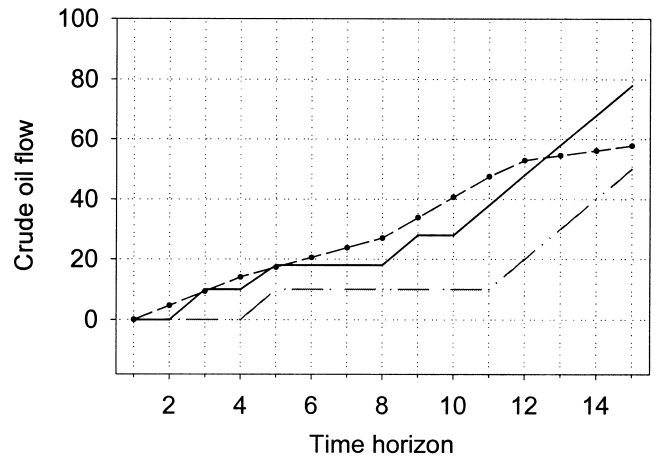

(d) Tracking reference trajectory from $\mathrm{BT}(3)$ to $\mathrm{CDU}(2)$

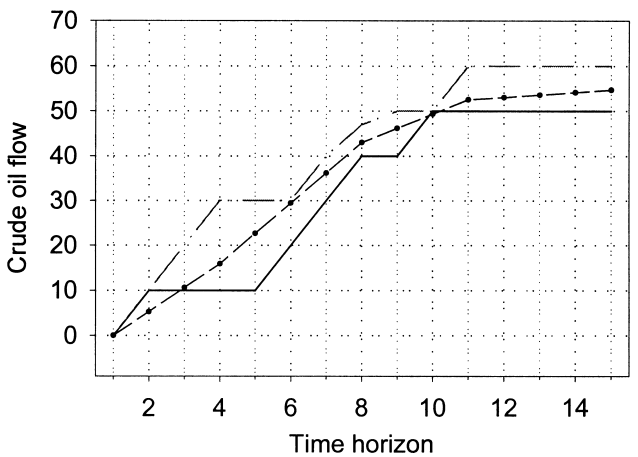

Fig. 10 Tracking reference trajectory from blending tank to CDU (example 2(c), (d)) 
(a) Tracking reference trajectory from BT(1) to CDU(1)

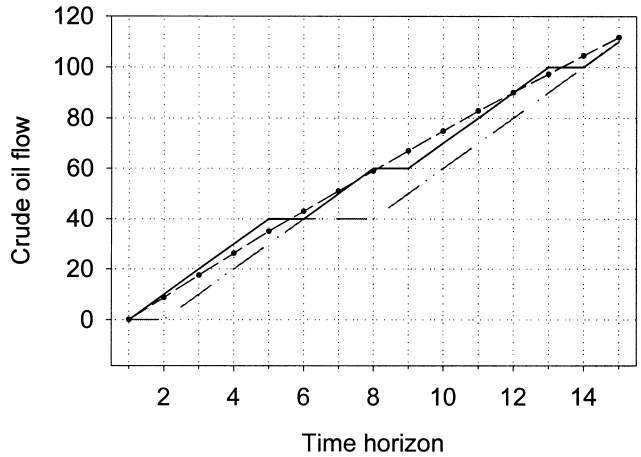

(b) Tracking reference trajectory from $\mathrm{BT}(2)$ to $\mathrm{CDU}(1)$

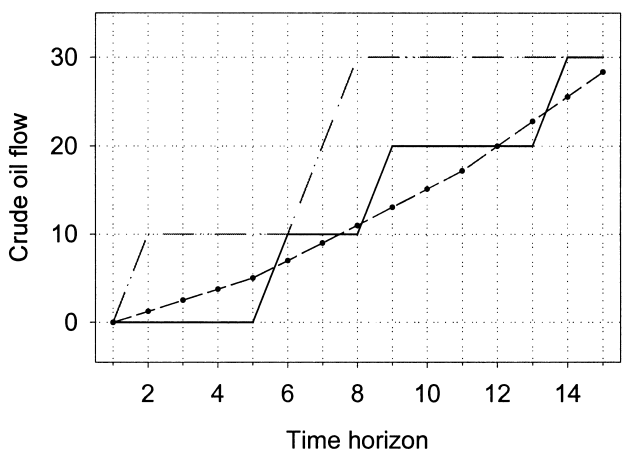

Fig. 11 Tracking reference trajectory from blending tank to CDU (example 3(a), (b))

(c) Tracking reference trajectory from $\mathrm{BT}(2)$ to $\mathrm{CDU}(2)$

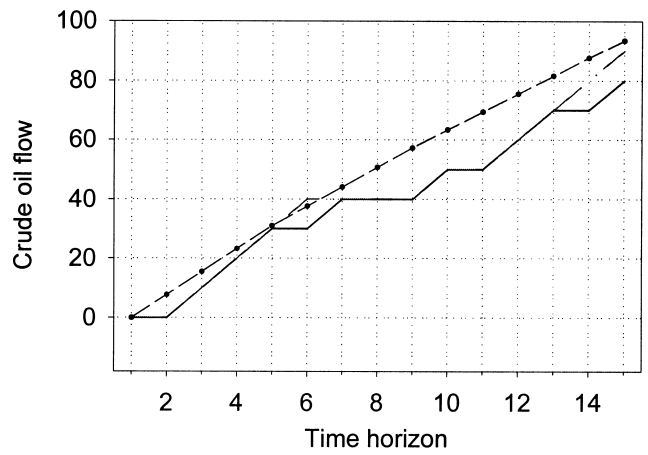

(d) Tracking reference trajectory from $\mathrm{BT}(3)$ to $\mathrm{CDU}(2)$

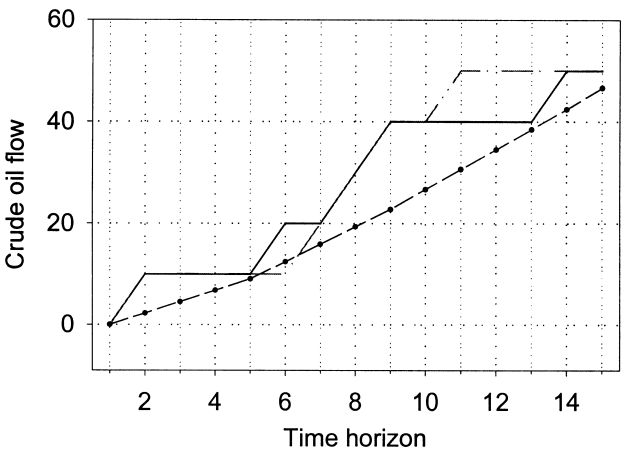

Fig. 12 Tracking reference trajectory from blending tank to CDU (example 3(c), (d)) (e) Tracking reference trajectory from $\mathrm{BT}(3)$ to $\mathrm{CDU}(3)$

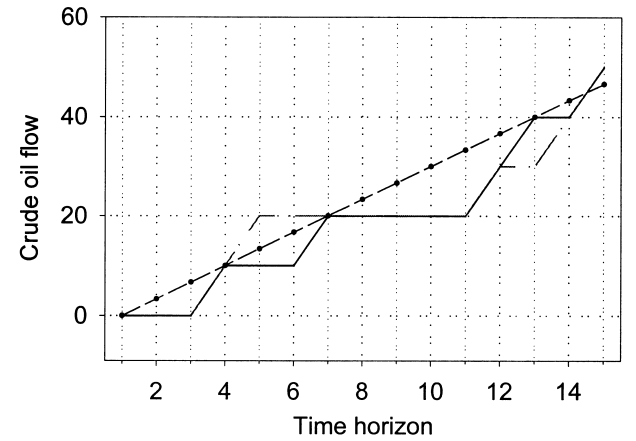

(f) Tracking reference trajectory from $\mathrm{BT}(4)$ to $\mathrm{CDU}(3)$

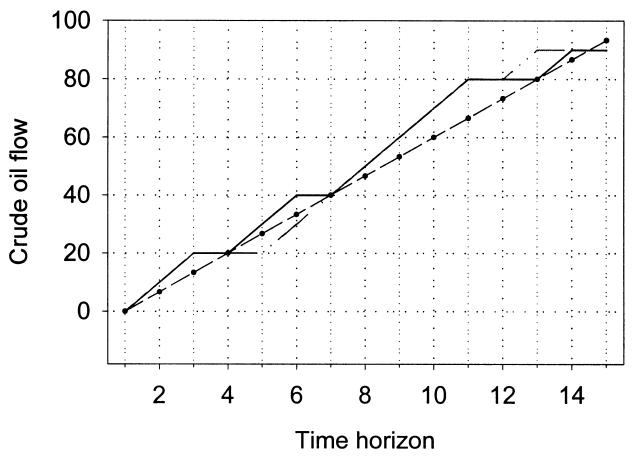

Fig. 13 Tracking reference trajectory from blending tank to CDU (example 3(e), (f))

antee the global optimum, the solution time is remarkably reduced.

\section{Conclusion}

The short-term scheduling problem arising in refinery processes has been addressed in this paper. A new scheduling algorithm based on the optimal control theory is proposed. The LP-based branch and bound method was applied to the model with two-level hierarchical method. Three examples of large-scale refinery processes are presented to show the performance of optimal control approach. While the proposed algorithm cannot always guarantee the global optimal solution, it reduces computational time by an order of magnitude as shown in the examples.

\section{Acknowledgment}

This work was supported in part by the Korea Science and Engineering Foundation (KOSEF) through the Automation Research Center at Pohang University of Science and Technology (POSTECH).

\section{Nomenclature}

$C_{\text {inv_ST, }, i}, \underset{\text { inv_BT }, j}{=}$

inventory cost of storage tank $i$ and inventory cost of blending tank $j$ per unit time interval

$C_{\text {setup }, j j^{\prime}, l}=$ changeover cost for transition from crude mix $j$ to $j^{\prime}$ in CDU $l$ 


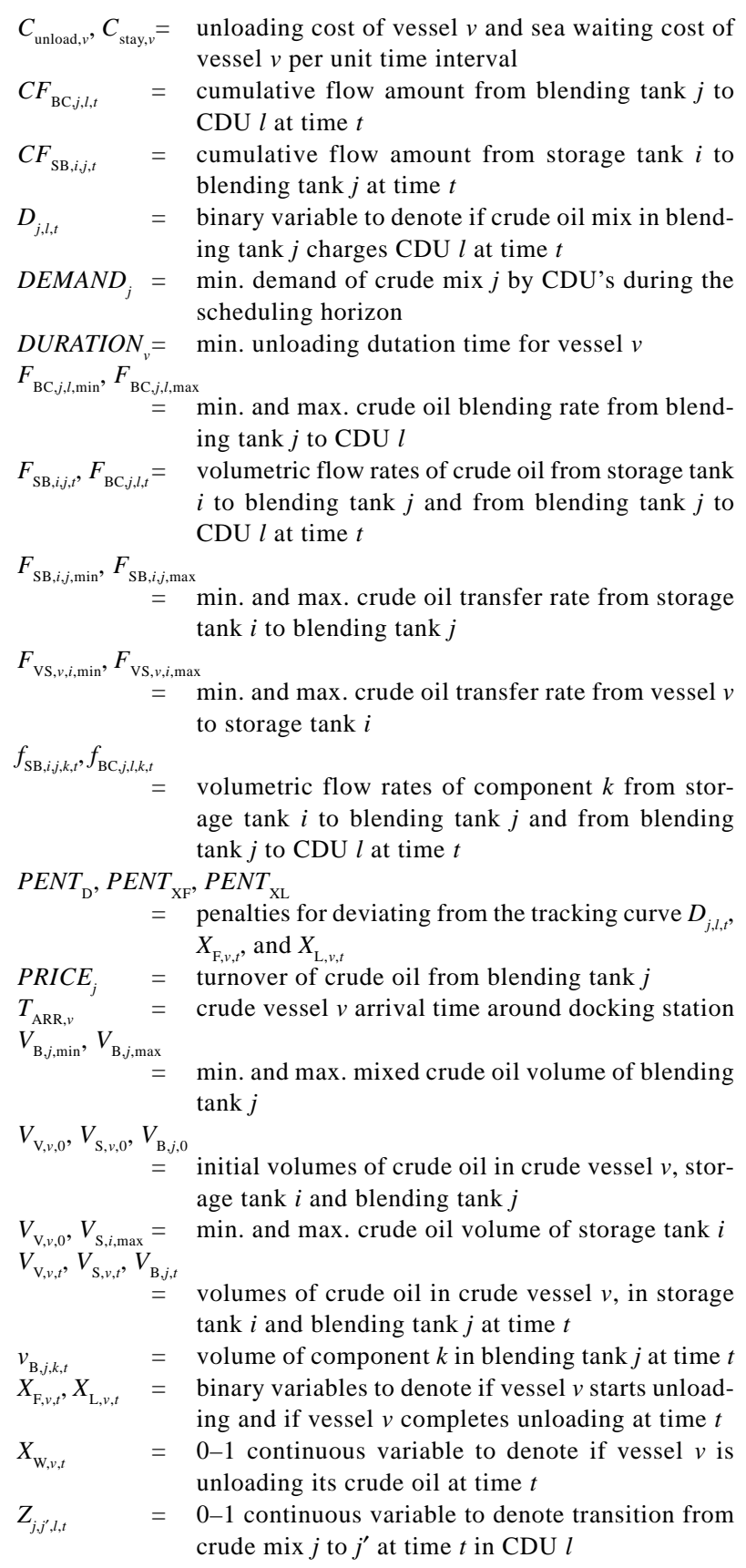

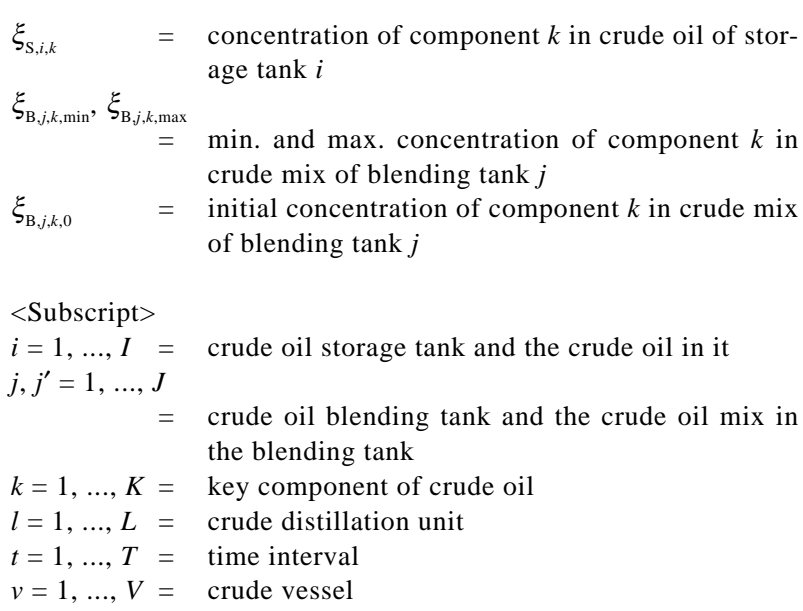

\section{Literature Cited}

Bok, J. and S. Park; "Continuous Time Modeling for Short-Term Scheduling of Multipurpose Pipeless Plants," Ind. Eng. Chem. Res., 37, 3652-3659 (1998)

Brandimarte, P., W. Ukovich and A. Villa; "Continuous Flow Models for Batch Manufacturing: A Basis for a Hierarchical Approach," Int. J. Prod. Res., 33, 1635-1660 (1995)

Brooke, A., D. Kendrick and A. Meeraus; GAMS: User's Manual, Scientific Press, Redwood City, CA, USA (1992)

Kogan, K. and E. Khmelnitsky; "An Optimal Control Model for Continuous Time Production and Setup Scheduling," Int. J. Prod. Res., 34, 715-725 (1996)

Lee, H., J. Pinto, I. Grossmann and S. Park; "Mixed-Integer Linear Programming Model for Refinery Short-Term Scheduling of Crude Oil Unloading with Inventory Management," Ind. Eng. Chem. Res. 35, 1630-1641 (1996)

Miltenburg, J.; "Level Schedules for Mixed-Model Assembly Lines in Just-In-Time Production Systems," Management Science, 35, 192-207 (1989)

Nott, H. P. and P. L. Lee; “An Optimal Control Approach for Scheduling Mixed Batch/Continuous Process Plants with Variable Cycle Time," Comp. Chem. Eng., 23, 907-917 (1999)

Shah, N.; "Mathematical Programming Techniques for Crude Oil Scheduling," Comp. Chem. Eng., 20, s1227-s1232 (1996)

Shah, N.; Single and Multisite Planning and Scheduling: Current Status and Future Challenges, FOCAPO, Snowbird, USA (1998) 